\title{
Ilang Tala Hinggil sa Narkotiko at Panganorin
}

\section{Allan Popa}

Ateneo de Manila University

\section{Abstrak}

Ang koleksiyong Narkotiko at Panganorin ay isang mahabang poetikong sunuran na pumapaksa sa mayamang pagpapakahulugan sa gabi sa kulturang Filipino. Tinitipon dito ang iba-ibang personal at pampublikong salaysay na nagpapakita ng dahas sa kamalayan ng pagkagambala ng mahimbing na pamamahinga dahil sa pribado at kolektibong ligalig. Isinasaakto ng mga tula kung paanong ang poetikong pamamaraan ay pangangapuhap din ng etikal na espasyo ng pakikipagkapwa sa gitna ng sigwa at pangamba. Ang pagsulat ng mga tula sa koleksiyong ito ay naging aralin mismo sa pagtuklas ng panlipunang dimensiyon ng pagtula. Kinailangan kong higit na maging malay sa pahina bilang espasyo at sa abot-tanaw sa paligid nito bilang sityo ng posibleng pakikipag-daupang palad sa kapwa. Ang pagtula, sa ganitong paraan ay hindi na lamang ekspresyon ng sarili kundi paraan din ng pagbibigay-puwang sa kalooban ng iba sa wika. Pagdadala ito sa sarili sa kalagayan ng kahandaang magambala o mapatahimik. Higit pa sa proyektong poetiko, naging pagkakataon ang pagsulat nito upang maipamalay sa akin na hindi lamang ako 
gumagawa ng tula kundi may ginagawa rin ang pagtula sa akin. Kung paanong makikita na walang pagkakaiba ang pagkakaanyo ng tula at ang anyo ng pakikipamuhay sa kapwa.

\section{Mga Susing Salita}

etika, gabi, kapwa, poetika, tula 


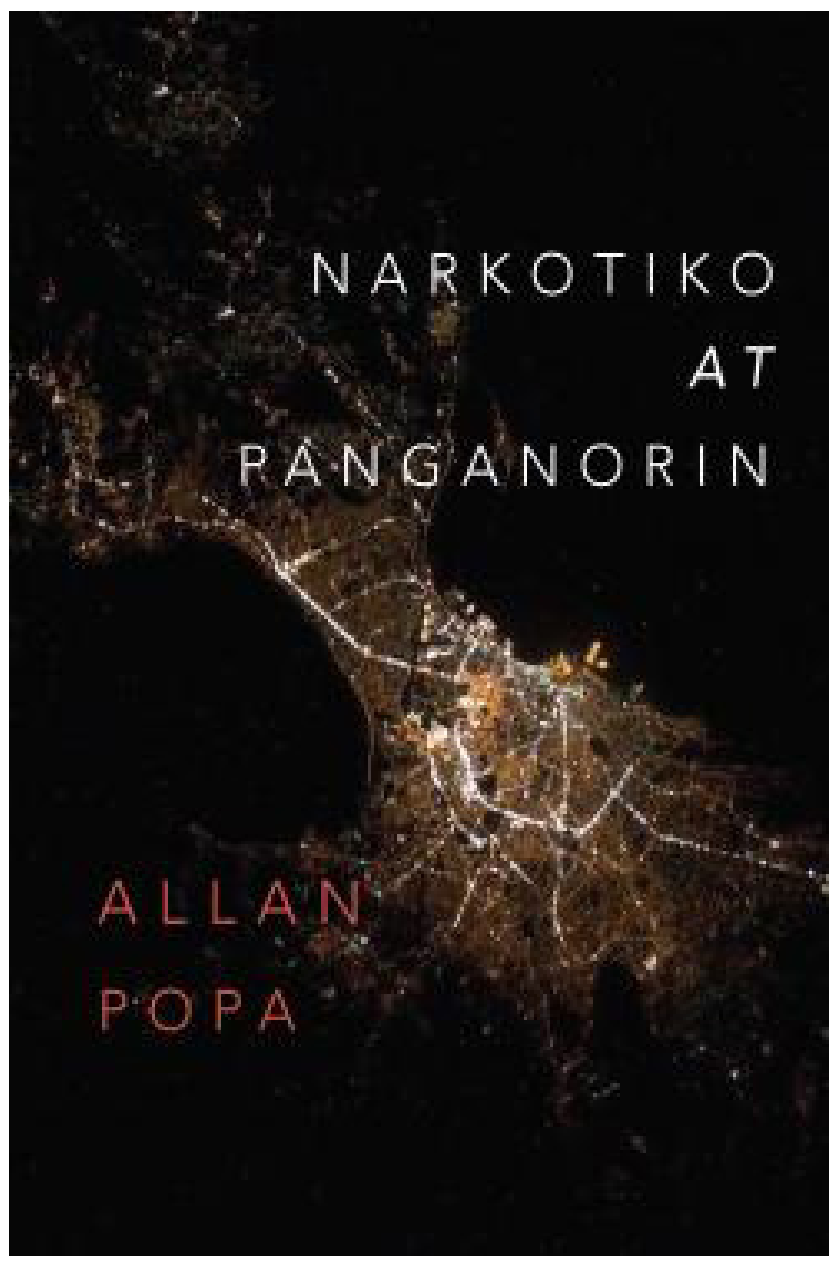

Fig. 1. Cover of Allan Popa's Narkotiko at Panganorin; http://www.ateneo.edu/ateneopress/ 
May pahayag ang Amerikanong makatang si Susan Stewart na naghawan ng landas ng proyektong Narkotiko at Panganorin. Mula ito sa aklat na Poetry and the Fate of the Senses na bumalangkas sa ilang susing kaisipan na nais ko ring talakayin sa mga tula. Napakayaman ng magkakasalungat na pagpapakahulugan sa gabi at dilim sa kultura at panitikang Filipino. Sa isang banda, nagbibigay rin ito ng kalinga at pahinga ngunit nagdudulot din ng matinding pangamba. Ang kalakhan ng kadiliman na bumabalot sa tao ay kapwa pinapaksa at puwersang nakadiin sa kamalayan na nagbigay-anyo sa mga tula.

The cultural, or form-giving, work of poetry is to counter the oblivion of darkness. To make such a statement may seem more fancifully "poetic" than true. But it is precisely in material ways that poetry is a force against effacement-not merely for individuals but for communities through time as well. The task of aesthetic production and reception in general is to make visible, tangible, and audible the figures of persons, whether such persons are expressing the particulars of sense impressions or the abstractions of reason or the many ways such particulars and abstractions enter into relations with one another. As metered language, language that retains and projects the force of individual sense experience and yet reaches toward intersubjective meaning, poetry sustains and transforms the threshold between individual and social existence (Stewart 2).

Naging higit na malinaw rin ang layunin ng koleksiyon nang mabasa ko ang sinabi ni Allen Grossman ukol sa gawaing kultural ng tula. Sa kanyang pagbibigay-katuturan sa tula, hindi ang mga pampanitikang aspekto nito ang binigyang-pansin niya kundi ang naisasaaktong paninindigan ng tula laban sa paglaho upang maikintal sa alaala ang hulagway ng pagkatao. Sa tula, nagkakatanawan ang mga tao sa magkakaibang panahon at lugar, kung saan maaaring magkadaupang-palad ang manunulat at mambabasa, ang kapwa, at ang iba upang alalahanin ang isa't isa.

Poetry is a principle of power invoked by all of us against our vanishing. The making of poems is a practice-a work human beings can do-in which civilization has invested some part of its love of itself and the world. The poem is a trace of the will of all persons to be known and to make known and, therefore, to be at all. Insofar as love wills the existence of what it loves, the principle of poetry is a collective and perpetually renewed act 
of love that brings the world to mind, and mind to mind, as the speech of a person-at the moment of the vanishing of world and persons, which is every moment of conscious life. Poetry is one means by which human beings engage, as they can, in the maintenance of a human world in which they can meet one another, affirm one another, remember, see, and foresee one another (Grossman ix).

Bago ko pa man simulan ang proyekto, nilinaw ko na sa sarili ang ilang bagay. Alam kong kailangang magsilbing sanggalang ang moda ng pagtula na mapipili ko sa materyal na lubhang personal at napakalapit sa akin upang makita ang mga limitasyon nito at mapalawak pa ang saklaw. Nais kong matiwalag sa karanasan sa pamamagitan ng anyo upang mas mahimay ang mga puwersang kumikilos dito.

Malinaw sa akin na nais kong magsulat ng mga tulang sumusuri sa mapaniil na mga puwersang panlipunan na pumupuno sa gabi ng sari-saring ligalig. Higit na publiko ang larangan ng mga tulang nais kong sulatin. Pakikilusin ko sa mga taludtod ang puwersang moral. Nais kong paganahin dito kung ano ang iniwasan ko sa mga nauna kong koleksiyon. Pakiramdam ko, hinihingi ng mga tula rito ang dulog na mas didaktiko upang magkabisa. Ayon nga kay Oren Izenberg sa sanaysay na "Poetry in and out of School":

Poetry, if it is to have any value, must contribute to our capacity to bear conditions of being that are as inescapable and as unresponsive to choice as the force of feeling in one's own heart - "to obtain for everybody one kind of success at the limits of the autonomy of the will." Among those limits are mortality, the solitude of the mind's private experience, the violence of history, and the blankness of a bare object world (192).

Ang magbigay-lugod at magturo-ito ang tinatanggap na dalawang pangunahing layunin na umiiral sa tula. Ngunit hindi maikakailang sa kasalukuyang estetikong panlasa, lalo na ng mga pinalalaganap ng kawal ng sining ng pagtula, mayroong pagdududa sa isa sa mga itinuturing na bisa nito: ang magturo ng aral. Kung pakikinggan ang mga puna at payo sa mga kabataaang manunulat, madalas sabihin ng mga eksperto: "Iwasang maging didaktiko." Masasabing nagmumula ito sa akalang inaagawan ng didaktikong 
tula ang mambabasa ng layang magpasya para sa sarili. Tila ba sinasabi na sumasalungat sa lugod na hatid ng sining ang kagustuhang magpaunawa ng aral. Pinuna ito ni Oren Izenberg:

If the delight of poetry was once seen as "the first light-giver to ignorance," a privileged vehicle and prerequisite for the preservation and transmission of other "tougher" forms of knowledge, then our present moment regards the pedagogical imperative in art more suspiciously: as a dulling of our pleasures, as a descent from art's higher calling, or as laying bare its bullying or "palpable design" upon us. The terms of art for poetry's traditional pedagogical genres and modes-sententious, moral, rhetorical, satiric-have been transformed into terms of abuse by generations of critics moved by Romantics' "abhorrence" for the didactic (Shelley), their preference for power over knowledge for "the tigers of wrath" over "the horses of instruction" (187).

May mahabang tradisyon sa ating kultura ang ganitong moda ng pagtula. Mula pa sa mga salawikain, mga naitalang pinakamaagang halimbawa ng maikling tula sa Vocabulario de la Lengua Tagala, mga tulang relihiyoso, mga tulang romantiko, at moralistiko, hanggang sa mga tulang propagandista at rebolusyonaryo. Naging napakahalaga ng ganitong uri ng tula sa pagpahayag ng saligang mga pagpapahalaga na naging pundasyon ng ideya natin ng bayan. Sa ganitong mga tula, ginamit ang poetikong pamamaraan upang mas maipadama ang mga kaisipang nais ipaunawa. Madalas na gamitin ang nalilikhang ambigwidad upang mapadanas sa mambabasa ang isang suliranin at matulungan siyang magpasya na pumanig sa pinaniniwalaang totoo at wasto batay sa kolektibong pagpapahalaga. Higit na rasyonal ang kilos ng isipan sa mga tulang didaktiko at may layuning mapanumbalik ang ipinapalagay na konsepto ng kaayusan. Madalas na nasa tinig ng awtoridad ang nagsasalita sa mga tula. Nais kong magamit ang ilang aspekto ng ganitong moda pero sa paraang may paggalang pa rin sa ahensiya ng mambabasa na magsuri at magpasya para sa sarili. Paano ba ito magagawa habang binubuwag din ang posisyon ng awtoridad? Kailangan ko ng distansiya ng perspektiba.

Sa simula, inakala kong dokumentaryong pagtula ang angkop na dulog kaya't napakaraming kaugnay na materyal mula sa iba-ibang larangan ang 
binasa ko at sinaliksik. Umasa ako na makahahanap ng estruktura kung saan maihahanay ang mga interesanteng kaalaman hinggil sa paksa upang mapalalim ang pag-unawa rito. Nagmumula ang udyok na ito sa kagustuhang sumubok ng naiibang estilo sa pagtula na malayong-malayo sa nakasanayan Para sa akin, isa sa mga nakasasabik na hamon sa kontemporaneong pagtula ay ang makahanap ng poetikong danas maging sa wika ng mga larangang hindi inaakalang magtataglay nito. Nakasasabik ang ganitong posibilidad pero hindi ko narinig ang sarili sa bunton ng wika ng pananaliksik at katunayan. Hindi ito gaanong nangusap sa akin ilang ulit man akong sumubok ng bagong kombinasyon at kompigurasyon ng mga sipi. Walang nalilikhang sapat na enerhiya ang banggaan o ugnayan sa pagi-pagitan. Walang napukaw na hiwaga.

Kaya naman naging mahalaga sa akin ang mga aklat ng makatang si George Oppen sa pagsulat ng manuskritong ito. Nabigyan niya ako ng halimbawa kung paano magpatuloy. Kung paano hahawakan na may sapat na layo ang materyal. Nakatulong ang pagsusuri ng makatang si Louise Glück sa akin sa pag-unawa sa bisa ng panulat ni Oppen:

Moral passion usually manifests itself in decisiveness, which becomes a compulsion to take sides. But a mind either intensely religious or unusually open may invest such passion in acts of speculation. These poems speak a moral language, a language of salvation and contempt. They have the force of true passion, but none of the smarmy definitiveness, none of the self-righteousness. Their beauty has always seemed to me the beauty of logic, the 'virtue of the mind' whose end is vision (9).

Sa pagbabasa ko sa mga aklat ni George Oppen, partikular na ang This In Which at Of Being Numerous, nahihikayat ako na pahalagahan ang katahimikan sa pagi-pagitan ng mga taludtod na kasimbigat din ng mga salita. Napawawari sa akin ang mga puwang kung saan nananahan ang pagkatula ng tula-sa mga bitak ng tula dumadaloy ang puwersa ng tula. Bilang mambabasa, inilulugar niya ako sa posisyon bilang tagatanggap ng puwersa ngunit hindi pasibong tagatanggap. Ang pagpapadaloy ng puwersang ito ay nangangailangan ng aktibo kong pag-iisip at pagdarama, ng pag-uugnay at 
pagpansin sa kaibahan. Kailangan nito ng aking presensiya at pinupukaw nito ang aking presensiya.

Masasabing ang tula ay lagi't laging nasa bingit ng pagkakabuo at pagkakalansag. Sa isip at pandama ko hinahawakan at pinakakawalan ang doble-karang katangian na ito. Para akong mala-barista na hindi maaaring hawakan nang ganap ang isang patalim dahil babagsak ang iba pa at masisira ang palabas. Kailangan kong matutuhan kung paano magkasabay na pakakawalan ang bawat hahawakan upang mapanatili itong nakalutangkung paano manahan sa kawalang-katiyakan. Ang bawat posibleng pagbasa sa pag-iilang-diwa ng tula, ang mga magkakatunggaling puwersa, ang mga balintuna at parikala ay isinasaalang-alang nang hindi kailangang magpasya kung alin ang tama. Naturuan ako nitong bumitiw, na hindi panghawakan nang labis ang pag-aari, na pahalagahan ang humigit-kumulang, at pagkahigit ng kakulangan.

Sa mga tula ni Oppen, nakahaharap ko ang isang kapwa na laging nirerebisa ang sarili, na nililitis ang sariling mga palagay, na may kakayahang gambalain ang sariling mga katiyahan. Para bang napapadanas sa akin lagi ang isang kamalayang nasa bingit ng pagbabago ng isip, puno ng pag-aalangan at pag-aatubili ang mga hakbang, puno ng kontradiksiyon. Hindi pa buo ang loob pero may mabigat na niloloob. Nararamdaman ko ang pakiwari niyang nakikiraan sa pahina dahil dama niya ang kapwa sa kaligiran ng tula. Dama niya rin ang aking presensiya.

Ang kamalayan na ito sa espasyo ng pahina bilang espasyo ng negosasyon sa pakikipag-ugnayan ay bunsod marahil ng karanasan ko sa pagtuturo ng panitikan sa unibersidad. Bilang guro na rin ng pagbasa at pagsulat ng tula, naging hamon na sa akin kung paano mapalalawak ang pag-iisip ng mga estudyante hinggil sa lugar ng tula sa lipunan. Hindi upang mahanapan ng praktikal na gamit sa buhay ng mga estudyante (naniniwala ako na tumututol ang tula sa mismong ideya ng pakikinabang) kundi upang makita nila ang ugnayan ng pagkakasantabi nito sa maaaring bisa ng sining na ito. Gusto kong mainitindihan nila kung bakit kailangang maging masalimuot ito.

Nakatutulong ang mga ideya ni Theodor Adorno na ipinahayag niya sa "Lyric Poetry and Society” na nagpaliwanag ng halaga ng pagiging-nasa-labas 
ng tula sa lipunan. Ang kahirapang naipadaranas ng tula sa mambabasa ay pagtutol ng tula na maipaloob sa kalakarang namamayani. Ang pagkatiwalag nito ay ang sityo ng politika na paninindigan at mapanuring pag-iisip ng tula na nakapaloob sa mismong anyo ito.

Ito ang pangunahing argumento ni James Longenbach sa kanyang aklat na The Resistance of Poetry. Para sa kanya, ang bisa ng tula ay nasa mga pagtutol nito na nakapaloob sa taludturan at iba pang pamamaraang ginamit. Tila ba ang mambabasa ay laging nananahan sa pagitan ng paghinto at pagpapatuloy na epektibong nakakasangkapan ng makata para mapawari ang mga suliraning pantaong inihahayag at isinasaakto rin nito. Ngunit ang pagtutol na ito ay di lamang pormal na estratehiya kundi paraan din ng pagkuwestiyon ng tula sa sarili nitong awtoridad.

Maaaring iugnay ang pananaw na ito ni Longenbach sa mga pangunahing kaisipan ni Allen Grossman na lalong naging impluwensiyal sa akin sa paglalatag ng mga bagong proyekto. Nang makaharap ko ang mga sanaysay sa aklat niyang The Long Schoolroom: Lessons in the Bitter Logic of the Poetic Principle, napamalay sa akin ang bisa ng kabiguan ng tula sa nais nitong magawa. Inuugat niya ang hanggahan na ito ng tula-ang puwang sa pagitan ng layon ng tula at sa kayang maisakatuparan sa wika-sa nakapaloob na kontradiksiyon sa kalikasan ng tula. Ayon sa kanya, ang sinusuri at tinutuligsa ng tula na mapaniil na sistema ay bahagi ng mismong kayarian ng tula na pinanday rin ng kasaysayan ng sining na ito at posibleng napalalaganap sa pamamagitan ng tula.

Nagdala sa akin ang ganitong mga pagpoproblema sa kalikasan ng tula sa pag-iisip hinggil sa panlipunang aspekto o gawain ng pakikipag-kapwa ng mga pamamaraang pampanitikan. Ano nga ba ang nagagawa ng imahen sa tula sa pagpapaigting ng pakiwari natin sa panahon? Sa mga gambalang dala ng mga putol ng linya, paano ba nagpapamalay ng salungatan ng kaisipan na kapwa totoo at hindi kailangang ipawalang-bisa ang isa't isa? Ano nga ba ang nagagawa ng kakayahan nating guni-gunihin ang suliranin ng isang persona sa kakayahan din nating maisip ang mga buhay na iba o malayo sa atin (sa lugar man o panahon)? Sa ganitong paraan, tila nabubura ang kaibahan ng mga papel na ginagampanan ko sa buhay bilang manunulat, mambabasa, 
mag-aaral, at guro ng tula dahil bahagi ang lahat ng aspektong ito ng pagiging mamamayan ko.

Nasasabi kong ang tula ay may puwersa ng tinig na nagsusumamong marinig kung napadarama nito sa akin na kailangan ako ng tula para ito mabuo. Para bang nasa hinagap na ako ng tula kahit pa hindi ito tungkol sa akin o para sa akin. Sa halip na sabihing nakauugnay ako sa tula, mas angkop na ilarawang nasasangkot ako sa tula. Panandaliang nabubuo ang loob ko sa tulang binabasa ko upang muling malansag. Panandalian ding nabubuo ang loob ko sa pagsulat ng tula bago muling malansag. Ang tula ay lagi't laging nasa pagitan ng pagkakabuo at pagkakalansag. Sabi nga ni Carl Phillips sa aklat na The Art of Daring:

We make of the fragments of self a form that holds, briefly-that's the poem-then we watch it shatter again-which is, I suppose, that space that the poem fooled us into believing we'd left behind us, for a time, world of fragmented selves, hard truths, glinting ambiguities to be negotiated, navigated through as we make our disoriented way forward, or what we have to believe is forward. Like being mapless in tough territory, and knowing, somewhere inside, we'd choose this life, and this one only, if in fact we could choose (72).

Malaki ang naituro ng ganitong paglulugar ng sarili bilang mambabasa sa proseso ko rin ng pagsusulat. Tinatawag ko itong etikal na pangangapuhap. Sa ganitong pagtula, itinuturing ko rin ang pahina bilang pampublikong espasyo kung saan nagtatagpo ang pribadong sarili at ang posibilidad ng publiko. May kaakibat itong negosasyon ng pakikipag-ugnay sa kapwa-kung saan ako nagtatapos at kung saan nagsisimula ang iba. Nagpapamalay ito ng ideya ng hanggahan hindi bilang hindi-matinag na linya kundi okasyon para mawari ang presensiya ng kapwa. Hinihingi nitong mabuksan ang isipan at pandama. Dinadala ako sa estadong ito ng binabasang mahusay na tula na kinikilala ang aking presensiya. Ito ang estado na kailangan ko rin upang makalikha. Ang pagkabukas ng isip at pandama. Ang pagtataya ng sarili ay pagbubukas ng sarili sa bigay ng mundo at sa kusang maibibigay mo sa anyo ng lilikhaing sining. Si Ann Lauterbach sa aklat na The Given and the Chosen: 
I was interested then, as I am now, in asking questions about the possible relation between forms of life and forms of art. At the time, I was concerned with individuals, persons, identities; I wanted to know if the sequence of choice, decision, and judgement in one's quotidian life might have some bearing on that same sequence, choice, decision, judgement, in making art. Was there some innate tendency or even habit in both domains that could be discerned, so that a work of art somehow captured the essence of a person? Contrarily, could the process of making choices, decisions, and judgements in art-making in fact condition and shape a person's life? Perhaps, unsurprisingly, the figure that came closest to resolving this conundrum for me was John Cage. In Cage, there seemed to be a continuum between the given and the chosen so closely calibrated as to be indistinguishable, seamless. One sense in Cage an active receptivity that reconfigured desire into a form of waiting or listening; that reset choice into operations of chance. Somehow, Cage had found a way to convert life into art and art into life; this seemed to me an emblem or personal contentment that might extend into the world as an ethical proposal (13).

Paano nga ba ilalagay ang sarili sa paraang bukas sa mga posibilidad? Paano itatrato ang pahina bilang larang ng potensya? Paano ba maihahanay ang pagkamalikhain sa kakayahang isaalang-alang ang kapwa? Paano patatalasin ang pandama sa presensiya ng iba na nasa ligid ng pahina? Paano mananahan sa kahandaang magambala ng iba? Paano ba itataya ang sarili sa puwersang maaaring magdala sa bingit ng pagbabago? Paano maghahawan sa wika ng danas na pupukaw sa isipan at pandama ng mambabasa? Paano maninindigan sa kahandaang matiwalag sa kinatatayuan? Sa ganitong paraan ko inuunawa ang kakayahan ng tulang magdiskaril at magdala sa hindi inaasahan. Nasa kahandaan nitong magbigay sa alteridad. Hindi ito pagsuko at sa halip, aktibong pagpapatalas ng pakiwari sa iba na tanda rin ng sariling presensiya.

Is that really, though, what a poet wants, an erasure of estrangement, speech between two people? Or is the effort . . . enough? Inadvertently, I think, we speak from our own hauntedness to the hauntedness in others, those readers with whom our private poem, as if magically, resonates-that stranger who, having read the poem, says: "I have been there, known that place, though I did not know it entirely, or not at least like this. And I have felt the same." But again, this resonance-which is a form of response-is at 
best the hoped-for but not exactly expected result of a dialogue that's inadvertent; it's not the reasons for writing, or it isn't for me, at least. I think I write not to understand struggle and to somehow by understanding it come to some sort of peace with it, but to understand just enough to know how much more there is, still, to be understood; not an end, then, to struggling, but a stepping more deeply into it (Phillip 69).

Ang piyesang "Wikain Mo" ang unang nasulat kong tula sa koleksiyong Narkotiko at Panganorin. Itinakda nito ang tinig na susundan ko sa pagsulat ng mga kasunod na tula. Inilalarawan dito ang proseso ng pakikinig sa wika ng tula na nag-uudyok ng tugon bagama't hindi talaga matukoy kung saan o kung kanino nagmula. Hindi rin alam dito kung saan patungo. Bulag ito sa pangangapuhap ng susunod na salita upang makausad. Tatanggapin nito ang kasangkapan na maiaalay ng mundo at magkakasya sa hubog ng kanyang kamay upang makapagpatuloy.

Nang marinig ko sa wakas ang mga salita na higit sa mga salita

Hindi, hindi wika kundi himig na marahan ang diin sa pandinig dahil walang bibig.

Nasabik ako nang "marinig" ang himig ng panimulang mga linya ng tulang ito dahil bago sa akin. May naipadama sa akin ang kibot sa pag-uulit na "salita/salita" at "Hindi, hindi." May partikular na diin ito sa pandinig na dama hanggang sa ubod ng kamalayan. Tila inilalarawan ng mga taludtod na ito ang sariling pagdating sa aking kamalayan. Napakarahan pero may pagsusumamo ang tono. Napakapayak, napakaliliit ng mga salita. Wala halos imahen, ngunit ang tono, taglay ng tono ang pinagdaanan ng tinig. Waring naapuhap ko ito sa guho. Parang may guwang sa loob. Naiparinig nito ang katahimikan bago namutawi ang unang salita. Naiparinig ito ng katahimikan. Ramdam na ramdam ko ang diin sa tagpuan ng salita at katahimikan. 
Nang itala ko sa papel ang mga salita, hiningi nito ang maiikling linya na walang bantas. Maliliit na hakbang ng pag-usad. Marahan. Pero may pigil na bigat. May alinlangan ngunit bukas. Alam kong may nasimulan ako sa mga linyang ito ngunit hindi ko maaaring madaliin. Napakahina pa ng tinig. Hindi ko alam kung may mabubuong pangungusap. Kailangan kong maghintay. (Kung may malinaw na naituro ito sa akin, ito ay ang sining ng paghihintay.) May kutob ako na may maipauunawa dahil may nais itong maunawaan. Kung susundan ko lang. Kung hahayaan.

May magagawa ang pagpapaubaya. Tila ito maingat na paghahanay ng mga bato upang maanyayahan ang tubig na dumaloy. Bagama't wala kang hawak kundi mumunting salita. Hindi tubig ang hinihintay na dumaloy ngunit may kakayahan ding magtighaw ng malalim na uhaw kung mapadadaloy. Kung hahayaan ko itong baguhin ako, kung handa akong mabago, magbabago ang tungo nito.

\footnotetext{
May lugod na nadama

sa pag-aantala

at pagtitig sa mga kamay

na nasa bingit ng

pag-abot sa katam kung

karpintero.
}

Nang maidugtong ko ang mga linyang ito, tila may mali na narinig ko bilang tama. Alam ko na noon na mayroon akong natumbok na paraan ng pagpapatuloy. Natuto akong manahan sa kawalang-alam. At magtiwala. May karunungang taglay ang wikang ito. Alam kong may magagawa ang bagong wika na ito sa loob ng wikang alam ko. Alam kong may magagawa ako sa wikang ito. Sabi ni Robert Frost sa sanaysay na “The Figure a Poem Makes,” kailangang magpadala sa sariling tunaw.

For myself the originality need be no more than the freshness of a poem run in the way I have described: from delight to wisdom. The figure is the same as for love. Like a piece of ice on a hot stove the poem must ride on its own melting. A poem may be worked over once it is in being, but may not 
be worried into being. Its most precious quality will remain its having run itself and carried away the poet with it (132).

Tila kayang gambalain ang anumang katiyakan ng tonong iyon. Tila makapagdidiskaril ng pahayag upang dalhin ang kaisipan sa hindi inaasahang kabatiran. May pangako na makapagbubukas sa marahang paraan ng nagkukubli sa rabaw. May dalang kalinawan ang mga taludtod na iyon. May kagaanan na maaaring magpakilos ng bigat ng nais kong masabi. May tiwala ako na may mapasasaling itong damdamin kahit na gumamit ng abstraksiyon.

Lungsod ang lunan ng karamihan sa mga tula. Mga kalye. Paupahang mga silid ng gusali. Makasaysayang baluwarte sa gitna ng Maynila. Pagawaan. Nangangayayat na baka sa bangketa. Bahay-ampunan. Sanglaan. Bangko. Jeepney. Ospital. Sinehan. Paliparan. Telebisyon. Radyo. Hindi matulaing mga lugar. Lunan ng prosa ng araw-araw. Iba't ibang tauhan. Iba't ibang salaysay. May nasaksihan. May nasaliksik. May nakuwento lang. May nabasa sa nobela. May napanood. May napakinggan. Maraming bata. Naparaming mga bata.

Ang tinumbasan ko ng mga salita ay pakiramdam na nasa himig, hindi ang kaisipan. Ngunit naipadadaloy ang kaisipan. Sinundan ko ang tulak ng pangangailangan. May kakayahang bumigay sa diin ang mga taludtod na ito. Nagkakauka ang wika hindi dahil sa diin mula sa labas kundi dahil sa kabig mula sa loob na dala ng matalas na pakikiramdam sa abot-tanaw ng pahina, sa abot-dinig ng kamalayan. Kakaiba ang naging pagdaloy ng mga taludtod. Pagdaloy sa kabila ng alinlangan, pagdududa, pag-aatubili ngunit itinutulak ng kagustuhang magwasto sa kamalian. Hinahayaan kong mabago ang tinutungo nito. Lagi kong ginagambala. Sinusubok kung magagambala. Nagagambala ako kasabay ng pagkagambala ng wika. Hindi pagkamatatas kundi pagkautal. Iyong kalinawan na nasa dulo ng dila na hindi maiwika.

Napatalas ng pagsulat ng mga tula sa koleksiyong ito ang kakayahan kong makinig. Ang pakikinig, kung matututong makinig ay pakikiniig. Ngunit sino nga ba ang pinakikinggan sa katahimikan? Ano nga ba ang sinikap marinig? Ang dinidinig ko ay taimtim na pakikinig. Ang pinakikinggan ay ang ipinapalagay na nakikinig sa katahimikan. Naghihintay. Kung gagambalain ko ang 
pakikinig na ito, anong sasabihin ko? Sasabihin ko ba ang hinihintay niyang marinig? Ang kamalayan sa posibleng makaririnig ay may puwersang pumipigil sa mga salitang bibitawan. May kakayahan itong baguhin ang daloy ng pahayag. Ang tula ay nagkakaanyo sa pagitan ng dalawang pakikinig.

Ako ba ang nagsasalita sa mga tula? Tinig ko ba ang narinig at pinakinggan? Hindi ito ang tinig ko na naririnig kapag nagsasalita. Tinig ba ito ng isipan? Tinig ng kalooban? Ano ang kaugnayan ng tinig na ito sa pagsasalita ko? Ito ba ang sarili kong kaibahan? Hindi ko masabi. Ang alam ko lang, binibigyan ako ng tonong taglay ng tinig ng sapat na agwat kung saan ako tatayo. May paa ang tinig (kahit walang katawan). Hindi tunay na paa kundi pakiramdam na may paninindigan. May kinatatayuan sa lupa. Hindi nakalutang. Ako na hindi ako. Ako na nagsasaalang-alang sa iba. Ako bilang iba. Ako na kailangang manahimik upang marinig. Ramdam ko ang layo. Ramdam ko ang kailangang tawirin. Bakit kailangang bigyang-diin ang layo? Dahil hindi tiyak kung makararating. Wala talagang bisa kung tutuusin. Walang nagagawa kundi pukawin ang pakiwari. Hindi upang makapangyari. Hindi upang magbigay ng kalutasan. Kundi upang maiangat ang hintuturo.

Binitbit ko ang tinig na ito. Pinangalagaan sa isip. Naglakbay ako. Binitbit ko ang kalagayan, ang mga palagay, at pakikipagpalagayan. Binitbit ko ang mga talaan at panulat. Binitbit ko ang mga sinusulat. Binitbit ko ang hindi ko pa naisusulat. Binitbit ko ang aking pag-iisa saanman magpunta. Binitbit ko ang katahimikang hinuhubog ng aking silid. Binitbit ko ang aking silid. Nakapaligid ito sa akin. Kung gaano katalas ang ligid na ito ng pakikiramdam. Kung gaano kahandang tumugon. Kung gaano kabukas tumanggap ng wala sa hinagap. Kung gaano kabukas sa estranghero. Kung gaano kahandang mabago. Kung gaano kahandang malagos o maging lagusan. Ito ang aking ahensiya. Ito ang aking pagkukusa. Ito ang aking pagpapaubaya. 


\section{Sanggunian}

Adorno, Theodor. Notes to Literature: Volume I. Translated by Shierry Weber Nicholson, Columbia UP, 1958.

Frost, Robert. "The Figure a Poem Makes." The Collected Prose of Robert Frost, edited by Mark Richardson, Harvard UP, 2007, p. 132.

Glück, Louise. Proofs and Theories. Ecco P, 1994.

Grossman, Allen. The Sighted Singer: Two Works on Poetry for Readers and Writers. Johns Hopkins UP, 1991.

Izenberg, Oren. "Poets in and out of School: Allen Grossman and Susan Howe." The Cambridge Companion to American Poetry Since 1945, edited by Jennifer Ashton, Cambridge UP, 2013, pp. 187-201.

Lauterbach, Ann. The Given and the Chosen. Omnidawn Publishing, 2011.

Longenbach, James. The Resistance to Poetry. U of Chicago P, 2004.

Phillips, Carl. The Art of Daring: Risk, Restlessness, Imagination. Graywolf P, 2014.

Stewart, Susan. Poetry and the Fate of the Senses. U of Chicago P, 2002. 
Mga Tula mula sa Koleksiyon

WIKAIN MO

Nang marinig ko sa wakas

ang mga salita na higit

sa mga salita

Hindi, hindi wika kundi

himig na marahan

ang diin sa pandinig

dahil walang bibig

May lugod na nadama

sa pag-aantala

at pagtitig sa mga kamay

na nasa bingit ng

pag-abot sa katam kung

karpintero

Ang martilyo ay martilyo

hangga't hindi nahahawakan

ang sarili sa sariling init

Pero ang tinig

na nakaaantig, nakatatagpo

hindi man patuluyin

ng hindi alam na siya

ay naghihintay

sa hindi alam. 


\section{LIWANAG AT DILIM}

May oras kung kailan

higit na malaki

sa mga bata

ang kanilang anino.

Hayaan sila sa labas

sa sirkulo ng kanilang laro

habang pilit na

inaaninag sa pagdilim

ang nabuburang mga hanggahan

na binakas ng mga daliri

dahil nariyan na bago pa sila

tumuntong sa lupa.

Ang paglahuin ang mundo

ang layon ng laro

upang walang malabi sa isip

kundi alituntunin

at mga guhit na naghahati

lagi sa dalawang panig.

Walang ibang

makapagtuturo

kundi ang takipsilim

upang mamulat ang katawan

sa paglawak ng mga hadlang

tungo sa paglaho

at pagbitiw sa kamay ng kalaro. 
SA LILIM NG KULAMBO

Kung pagmamasdan

ang mundo mula

sa loob ng kulambo

higit tayong magiging

mapagpatawad.

Kung hindi tuwiran

ang pagbubunyag ng liwanag

sa mga pagkukulang.

Bigyan ang isa't isa

ng distansiya ng

punto de bistang

hindi nakaaalam ng lahat.

Kay gaan ng laylayan

na laging nasa bingit

na hawiin tulad ng belo

para masilayan

o mahagkan.

Ngunit hahayaan

ang kalabuan sa pagitan

ng pagdapo ng antok

at ng lamok.

Tandaan: hindi ito lambat

na panghuli ng panaginip.

Bigyan ng sapat

na anino ang mukha,

sapat na mga tuping

mapagkukublihan

ng alinlangan.

Walang hiwaga

ang gabi, ang hiwaga

ay may apat na pako

sa mundo na kinatatalian

ng kulambo. 


\section{RADYO}

Kasama natin sila sa paglagos

dahil lagusan ang lungsod

na nagbibigay-

pakiwari ng pag-unlad

maging sa naiiwan ng pag-unlad,

kasama sa pag-aabang sa kanto

sa pagtatapos ng araw

ang mga hindi pinatulog

ng sariling pag-aabang

na makatulog.

Para sa mga parusa

ang gabi

ang paglalapit ng tenga

sa radyo ay hindi nalalayo

sa paglapit ng bibig

sa dilim ng mga butas ng telepono

upang may maibulong

sa handang makinig.

Gasa para sa sugat

ang malumanay na sagot

ang payo

na hindi para sa akin

ngunit akin din.

Nanimbang ang mga daliri ko

sa tinig sa talapihitan.

May bagal ang pananalita

na nagsasabing

hindi kita iniiwan

kahit hindi mo na ako naririnig.

Patay na si Kuya Cesar, patay na si Tiya Dely.

Higit na tahimik

ang gabi ng libo-libong tagapakinig

na patuloy sa pakikinig. 


\section{JOHNNY MIDNIGHT}

Isa ang nanay ko sa kanila, sa mga gumigising bago maghatinggabi sa simula ng Dekada 80 para buksan ang radyo at iparinig sa tubig ang tagulaylay na nanginginig.

Wika ito ng pananampalataya na walang nakauunawa sa panahong walang makapitan kundi pananampalataya at tubig.

Alam ng mga ina.

May karamdaman na hindi matukoy sa katawan ngunit malinaw na nariyan dahil nabibigo ang mga salita na pangalanan.

Tinatanong niya ang bata sa kanyang bisig kung saan ang masakit ngunit wala itong maisagot kundi iyak nang iyak.

Walang pumupuno sa mga nakangangang sisidlan kundi matining na tubig. Maiinom mo ang sariling tinig. 